\title{
Glaucoma agudo bilateral por uso de topiramato: relato de caso
}

\author{
Topiramate-induced bilateral acute glaucoma: case report \\ Mardônio Parente de Menezes ${ }^{1}$
}

\section{RESUMO}

O topiramato, uma sulfa monossacarídica, usada primariamente como um anticonvulsivante, é uma droga relativamente nova no mercado brasileiro. Devido às potencialidades terapêuticas do topiramato em uma gama variada de patologias (enxaquecas, transtornos do humor, etc.), assim como pelas diversas novas apresentações comerciais surgidas no país ultimamente, espera-se que seu uso venha a ser cada dia mais comum. O presente

\section{Palavras-chave}

Topiramato, efeitos

colaterais, glaucoma agudo

bilateral. relato se refere a um grave efeito colateral decorrente do uso da medicação em questão, ocorrido em um homem de 36 anos - o glaucoma agudo bilateral, evento ainda pouco relatado na literatura mundial. Por conta de tal quadro, o paciente teve de se submeter a uma iridotomia bilateral.

\begin{abstract}
The topiramate, a monosaccharide sulfa, primarily used as an anticonvulsant, was recently introduced in Brazilian trade. Through therapeutic potentialities of topiramate in a considerable scale of illness (migraine, disorders of humor, etc.), thus by the recent presentation, in Brazil, of several news commercial presentation of medication, it is expected that its use be more common each day. This present study reports a severe side effect that comes from the use of the drug in evidence, occurred in a 36 year-old man - the bilateral acute glaucoma, event of report not usual in the world literature. Because of this event, the patient had to be submitted to a bilateral iridotomy.
\end{abstract}

\section{Keywords}

Topiramate, side effects, bilateral acute glaucoma. dores neuropáticas, dependência alcoólica', enxaquecas², transtornos do humor ${ }^{3}$, etc.

Pelas potencialidades terapêuticas do topiramato em uma gama considerável de afeç̧ões, assim como pelo recente lançamento, no Brasil, de diversas novas apresentações comerciais da medicação, espera-se que seu uso seja cada dia mais comum. Assim, efeitos colaterais antes tidos como raros podem ser cada vez mais frequentes na prática clínica.

1 Hospital de Referência de Porto Nacional, Tocantins, Divisão de Psiquiatria.

Recebido em

17/7/2009

Aprovado em

$18 / 8 / 2009$
Endereço para correspondência: Mardônio Parente de Menezes Quadra 706-Sul, Alameda 08, Lote 35 - 77022-386 - Palmas, T0 Telefones: (63) 3214-4775 / (63) 3228-6094

E-mail:mardonioparente@gmail.com 
Efeitos adversos decorrentes do uso do topiramato são relatados, na literatura, com uma frequência variável, sendo que, segundo alguns autores, tais efeitos podem levar à descontinuação do tratamento em $8 \%$ a $28 \%$ dos casos 4,5 . Os efeitos colaterais relatados vão desde alterações mais leves (náuseas, sedação, acidose metabólica, perda de peso e disartria) às mais sérias e preocupantes (nefrolitíase e glaucoma de ângulo aberto) ${ }^{6}$. Dentre os efeitos adversos relatados na literatura se incluem os de ordem neuropsiquiátrica, tais como: síndromes afetivas, psicoses, distúrbios de personalidade e de comportamento, além de encefalopatias?.

O presente trabalho tem por objetivo relatar um efeito colateral grave - o glaucoma agudo bilateral - decorrente do uso do anticonvulsivante em questão, evento de relato pouco frequente na literatura mundial.

\section{CASO CLÍNICO}

Paciente do sexo masculino, 36 anos, empresário, divorciado há 2 anos. Queixava-se, há 5 anos, de ansiedade, oscilações de humor, irritabilidade, adinamia, sonolência e aumento progressivo de peso. Apesar das oscilações do humor mencionadas, não havia - em seu relato - períodos com sintomatologia de ordem maníaca. Relatava ainda crises de cefaleia frontotemporal, bilateral, precedidas por escotomas e acompanhadas de fono e fotofobia, iniciadas anteriormente a suas queixas psiquiátricas. Portador de hipertensão arterial sistêmica, usava atenolol 50 mg/dia e clortalidona 12,5 $\mathrm{mg} / \mathrm{dia}$. Sem outras doenças. História familiar positiva para transtornos depressivos (avó e tia paternas) e para hipertensão arterial sistêmica. História de tratamento prévio com cloridrato de venlafaxina, na dose de 75 mg/dia, por 1 ano, com resposta questionável em seu humor. Por ocasião da primeira consulta, estava sem qualquer medicação psicotrópica há 14 meses. Exames solicitados (AST ${ }^{(1)}$, ALT $^{(2)}$, hemograma, glicemia de jejum, TSH$^{(3)}$ e T4-livre $\left.{ }^{(4)}\right)$ normais. Diante do quadro, formularam-se os diagnósticos de depressão atípica e enxaqueca.

Depois de um ensaio com moclobemida (até 600 mg/ dia), cuja resposta foi apenas parcial, o paciente passou a usar oxcarbazepina (até 900 mg/dia) em associação com cloridrato de trazodona (até 300 mg/dia). Em comparação com a moclobemida, houve melhora significativa do quadro de enxaqueca, assim como do quadro de humor.

Como viesse ganhando peso, o paciente suspendeu, por conta própria, a oxcarbazepina. Três semanas após a suspensão, foi-lhe prescrito topiramato ( $25 \mathrm{mg} / \mathrm{dia}$ e, após
4 dias, $50 \mathrm{mg} / \mathrm{dia}$ ) e foi mantido o cloridrato de trazodona em 300 mg/dia.

No segundo dia de uso do topiramato em $50 \mathrm{mg} / \mathrm{dia}$, o paciente procurou a emergência com um quadro súbito de dor ocular bilateral e deterioração repentina de sua acuidade visual, em ambos os olhos. Na emergência foi-lhe diagnosticado glaucoma agudo bilateral e o paciente foi encaminhado ao oftalmologista.

Ao primeiro exame, foi constatada - bilateralmente - íris periférica, ocluindo o ângulo camerular, além de pressão intraocular (PIO) de $58 \mathrm{mmHg}$, em ambos os olhos. Dois dias depois, com o tratamento clínico e a suspensão do topiramato, a PIO era de 27 e 28 mmHg (olho direito e esquerdo, respectivamente). Mesmo com melhora significativa, por indicação do oftalmologista que o assistiu, o paciente foi submetido a iridotomia, no dia seguinte ao dia em que deu entrada na emergência.

\section{DISCUSSÃO}

O topiramato é uma droga cujo mecanismo de ação está ainda por ser mais bem elucidado, contudo a atividade gabaérgica, a modulação dos canais de sódio voltagem-dependentes e o antagonismo glutamatérgico parecem fazer parte desse mecanismo ${ }^{8}$. Em comunicado oficial, datado de setembro de 2001, a US Food and Drug Administration (FDA) alertava para a possibilidade do desenvolvimento do glaucoma agudo bilateral em pacientes em uso do topiramato. Na ocasião, segundo aquele órgão, havia relatos de que, das 825.000 pessoas que fizeram uso da droga, 23 delas (sendo 22 adultos e 1 criança) haviam desenvolvido tal efeito colateral ${ }^{9}$. Pelo levantamento feito no presente trabalho, não há dados confiáveis para se saber a prevalência exata de tal efeito indesejável, contudo é razoável se supor que, com a maior atenção dada na literatura à questão, tais ocorrências sejam mais comuns do que se supunha em 2001.

Assim como é incerta a prevalência real do efeito adverso em questão neste relato, é também incerta a fisiopatologia a ele relacionada. A miopia aguda tem sido associada ao uso de diversas drogas derivadas da sulfa (por exemplo: acetazolamida, sulfametoxazol-trimetoprim, espironolactona, etc. ${ }^{10}$. No caso do topiramato, o estreitamento do ângulo iridocristalino poderia advir como consequência da diminuição da câmara ocular anterior. Tal diminuição seria ocasionada pela efusão uveal, que teria como consequência tanto o deslocamento anterior do diafragma iridocristalino, quanto o espessamento do próprio cristalino"1. Uma ação 
diminuída do anticonvulsivante sobre o inibidor da anidrase carbônica, assim como um efeito mediado por prostaglandinas, também têm sido postulados como possíveis causas do glaucoma agudo decorrente do uso do topiramato ${ }^{12}$.

Pelo pouco que há na literatura a respeito da fisiopatologia do glaucoma agudo bilateral decorrente do uso de topiramato, é difícil que se estabeleçam fatores de risco ou condições especificamente predisponentes para o desenvolvimento do efeito colateral aqui relatado. Uma grande variedade de condições pode vir a desencadear glaucomas secundários e, dentre elas, poder-se-iam citar: uveítes, traumatismos, anomalias congênitas e o uso de diferentes medicações ${ }^{13}$. Entre os pacientes que possuem o maior risco de desenvolver glaucoma secundário ao uso de medicações sistêmicas não esteroidais, estão: os portadores de hipermetropia, os portadores de câmara anterior rasa, pessoas com desproporção entre cristalino e segmento anterior, pessoas com ângulo iridocristalino estreito, além daqueles com olhos pequenos ${ }^{13}$. Enquanto se aguardam mais estudos que detalhem a fisiopatologia do glaucoma agudo bilateral decorrente do uso do topiramato, talvez tais dados nos possam servir, se não de guia, pelo menos de pistas que nos norteiem o uso mais atento e prudente da droga.

A grande maioria dos casos relatados na literatura, até o presente momento, obteve, com a suspensão do topiramato e com o tratamento clínico, remissão completa dentro de alguns dias. No caso presente, contudo, o profissional oftalmologista julgou necessária - quase que prontamente - a intervenção cirúrgica. O levantamento bibliográfico, empreendido por ocasião deste trabalho, é bastante claro em indicar o tratamento conservador, em caso de melhora com a suspensão do topiramato. O tratamento cirúrgico estaria reservado somente aos casos em que, após tratamento clínico, a PIO se mantivesse alta, fazendo com que houvesse um risco real de lesão ao nervo óptico"1.

A maioria dos casos relatados de glaucoma agudo decorrente do uso do topiramato ocorreu em pacientes do sexo feminino, sendo que também há casos relatados em crianças $^{14,15}$.

\section{CONCLUSÃO}

Por seu favorável perfil de efeitos colaterais, no que se relaciona ao ganho ponderal, o topiramato tem sido uma escoIha importante para pacientes que ganham peso com o uso de outras drogas psicotrópicas.
Este trabalho serve de alerta para que, na prática cotidiana, estejamos atentos ao efeito colateral relatado, já que, se não observado a tempo, poderá deixar sequelas irreversíveis", assim como levar a intervenções cirúrgicas desnecessárias.

\section{REFERÊNCIAS}

1. Castro LA, Couzi C. Uso potencial dos anticonvulsivantes no tratamento ambulatorial da dependência de álcool. J Bras Psiquiatr. 2006;55(3):212-217.

2. Krymchantowski AV, Tavares C, Penteado JC, Adriano M. Topiramato no tratamento preventivo da migrânea: experiência em um centro terciário. Arq Neuro-Psiquiatr. [periódico na Internet]. 2004 Mar [citado 2009 jul 17]; 62(1):91-95. Disponível em: http://www. scielo.br/scielo.php?script=sci_arttext\&pid=S0004-282X2004000100016\&lng=pt. doi: 10.1590/50004-282X2004000100016.

3. Moreno RA, Moreno DH, Soares MBM, Ratzke R. Anticonvulsivantes e antipsicóticos no tratamento do transtorno bipolar. Rev Bras Psiquiatr. [periódico na Internet]. [citado 2009 jul 17]. Disponível em: http://www.scielo.br/scielo.php?script=sci_arttext\&pid= S1516-44462004000700009\&lng=pt. doi: 10.1590/S1516-44462004000700009.

4. Ben-Menachem E, Henriksen O, Dam M, et al. Double-blind, placebo-controlled trial of topiramate as add-on therapy in patients with refractory partial seizures. Epilepsia. 1996;37:539-43.

5. Beghi E. Efficacy and tolerability of the new antiepileptic drugs: comparision of two recent guidelines. Lancet Neurol. 2004;3:618-21.

6. French JA, Kanner AM, Bautista J, et al. Efficacy and tolerability of the new antiepileptic drugs: I: Treatment of new onset epilepsy: report of the Therapeutics and Technology Assessment Subcommittee and Quality Standards Subcommittee of the American Academy of Neurology and the American Epilepsy Society Neurology. 2004;62(8):1252-60.

7. Schmitz B. Psychiatric syndromes related to antiepileptic drugs. Epilepsia. 1999;40(Supl 10): $565-70$.

8. Carvalho DF, Cercato C, Almeida MQ, Mancini MC, Halpern A. Abordagem terapêutica da obesidade na síndrome de Prader-Willi. Arq Bras Endocrinol Metab [periódico na Internet]. 2007 Ago [citado 2009 jul 17]; 51(6):913-919. Disponível em: http://www. scielo.br/scielo.php?script=sci_arttext\&pid=S0004-27302007000600004\&lng=pt. doi: 10.1590/50004-27302007000600004.

9. US Food and Drug Administration (FDA). Important drug warning. http://www.fda.gov/ medwatch/safety/2001/topamax.htm. Acessado em 8 de dezembro de 2008.

10. Chalam KV, Tillis T, Syed F, Agarwal S, Brar VS. Acute bilateral simultaneous angle closure glaucoma after topiramate administration: a case report. J Med Case Reports. 2008;2:1.

11. Marcon IM, Nunes CM, Taicher PH, Taicher F, Bergamini C, Thorell MR. Topiramato versus glaucoma agudo de ângulo fechado. Rev Bras Oftalmol. [periódico na Internet]. 2008 Fev [citado 2009 jul 17]; 67(1):39-41. Disponível em: http://www.scielo.br/ scielo.php?script=sci_arttext\&pid=S0034-72802008000100007\&lng=pt. doi: 10.1590/ S0034-72802008000100007.

12. Desai CM, Ramchandani SJ, Bhopale SG, Ramchandani SS. Acute myopia and angle closure caused by topiramate, a drug used for prophylaxis of migraine. Indian J Ophthalmol. 2006;54(3):195-7.

13. Almeida HG, Cohen R, editores. Glaucomas secundários. 2a ed. São Paulo: Roca; 2006.

14. Lachkar Y, Bouassida W. Drug-induced acute angle-closure glaucoma. Curr Opin Ophthalmol. 2007;18(2):129-33.

15. Levy J, Yagev R, Petrova A, Lifshitz T. Topiramate-induced bilateral angle-closure glaucoma. Can J Ophthalmol. 2006;41(2):221-5. 